\title{
Minimally invasive therapy of primary breast cancer
}

\section{David Robinson}

David S. Robinson M.D., "Minimally invasive therapy of primary breast cancer," Proc. SPIE 10297, Matching the Energy Source to the Clinical Need: A Critical Review, 102970C (24 January 2000); doi: 10.1117/12.375213

SPIE. Event: Digital Optical Computing, 1990, Los Angeles, United States 


\title{
Minimally Invasive Therapy of Primary Breast Cancer
}

\author{
David S. Robinson, M.D. \\ Director, The Breast Center \\ Saint Luke's Hospital of Kansas City \\ Kansas City, Missouri
}

Keywords: Breast Cancer, Temperature, Laser, Radiofrequency, Cryotherapy, Digital mammography, Stereotactic mammography, Magnetic resonance imaging, Ultrasound

\section{INTRODUCTION AND BACKGROUND}

Treating disease with little alteration has long been a goal of medical science. During the past quarter century, technological advances have brought forth minimally invasive approaches to the surgical diagnosis and treatment of cancer. In the domain of breast cancer, a less invasive sentinel lymph node biopsy may replace axillary lymphadenectomy for many patients, and image guided core biopsies have minimalized the degree of surgical intervention needed for tissue diagnosis. This mirrors the primary treatment of breast cancer that over the past century has progressed from mastectomy to breast preservation with a progressively diminishing operative field.

In 1895, when the first large series of radical mastectomies was presented, the breast, its underlying pectoral muscles, and the axillary contents were all removed en bloc. ${ }^{1}$ During the first half of the twentieth century, a decline in the negative stigma of cancer permitted patients to present earlier with smaller tumors, thus allowing for preservation of the anterior axillary skin fold and retention of the pectoralis major muscle (the modified radical mastectomy). Then in the 1960s, the advent of mammography began an era of discovery of small cancers before they became palpable, thus improving the chance for cure. ${ }^{2} \mathrm{By}$ the mid 1970s, both in the United States and in Europe, breast cancer was recognized early enough in its course that breast preservation following lumpectomy and radiation therapy became a reality. ${ }^{3}$ Lumpectomy followed by radiation has been prospectively compared with modified radical mastectomy in an aggregate of more than 3,000 patients in seven different clinical trials, demonstrating is no statistical difference in local recurrence and survival. ${ }^{4}$ This progressive decrease in the magnitude of resection without sacrificing cure is driven by the desire to diminish disfiguring change. A logical next step would be to design an internal treatment that would provide equal efficacy with minimal observable surface change, reduced patient trauma, and decreased overall cost.

Surveillance mammography of the asymptomatic patient now permits early enough discovery that survival rates in this country are improving. Very small breast cancers can be recognized and treated before they have an opportunity to metastasize. Using this technology, Blomgren and his colleagues demonstrated in the 1970s that two mammograms taken from a fixed point in the same curvilinear plane could accurately triangulate the position of a breast cancer in three dimensional space, creating a stereotactic approach for fine needle aspiration. ${ }^{5}$ In time, this progressed to core sampling for histologic analysis that

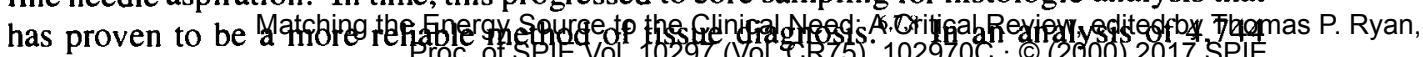


patients studied in twenty institutions, Parker $e$ al reported that cores taken by a 14 gauge needle stereotactically placed within one millimeter of the desired biopsy site have an accuracy equal to that of an open biopsy with needle localization. ${ }^{9}$ With the technological advance to digital mammography, stereotactic biopsy is gaining popularity because of the decreased time taken for such a biopsy. Today it is estimated that forty percent of image guided breast biopsies are performed stereotactically. This is essential for minimally invasive therapy. The patient with a surgical incision from an open biopsy following wire localization has already lost the advantage of subsequent minimally invasive interstitial therapy. Those who have been diagnosed by a small core biopsy are the beneficiaries of the coalescence of miniaturization, computer technology, and digital imaging that now permit minimally invasive therapy.

\section{DEMOGRAPHICS AND PATIENT VOLUME}

The last $1 \frac{1}{2}$ decades in this century have seen a remarkable increase not only in the quality of mammography but also in the numbers of patients examined. In 1985, the number of women in the United States who received mammography was estimated to be 7.5 million. In the year 2000 , that number is projected to be 48 million women. This remarkable increase reflects enhanced compliance with the recommended American Cancer Society/NIH guideline from 15 percent in 1985 to over 70 percent in 1998, all due to increased awareness of women in this country.

Current estimates suggest that two to three percent of women undergoing diagnostic mammography will come to biopsy. Based on that estimate, between 1 million and 1.25 million women will undergo an image directed biopsy in the United States this year, and the number undergoing stereotactic biopsy will represent a significant proportion (as high as $\mathbf{4 0}$ percent) of the total number of biopsies performed for non-palpable lesions.

Historically, 15 percent of image-guided breast biopsies demonstrate cancer. By that estimate, between 150,000 and 180,000 new cancers will be biopsied through needle localization or stereotactic biopsy. This estimate is high in light of a total estimate of 180,000 new cancers to be discovered this year in the United States. Still, if mammographically discovered cancers comprise only three quarters of the total number of breast cancers in the year 2000, then approximately 135,000 cancers will be discovered through an image directed biopsy. Assuming a conservative estimate of 40 percent undergoing stereotactic biopsy, more than 50,000 women with breast cancer will have been diagnosed by a core sample.

\section{BREAST CANCER BIOLOGY: SIZE AND TUMOR CLASSIFICATION}

An important consequence of greater surveillance and early diagnosis is a decrease in the mean size of the primary breast cancer at discovery. Cady et al have reported that the mean tumor size over seven decades has decreased from 3.5 centimeters to 1.5 centimeters. ${ }^{10}$ The authors suggest that this may approach a mean diameter of 1.0 centimeters within a short time. This tumor size is important to the development of prospective trials for interstitial therapy. Rosen et al report 10- and 20-year disease-free survivorship rates of 91 and 88 percent respectively in patients with $\mathrm{T}_{1 \mathrm{a}}-\mathrm{T}_{1 \mathrm{~b}}, \mathrm{~N}_{0} \mathrm{M}_{0}$ breast cancers ( $<10$ millimeters) treated 
by modified radical mastectomy. ${ }^{11}$ While the reason for this outcome has not been defined, we might suspect that tumors of this size are less likely to promote angiogenesis and lymphatic/vascular invasion.

With this in mind, Parker's series of stereotactic breast biopsies reported that 62 percent of all primary lesions, both benign and malignant, had a diameter of 10 millimeters or less. ${ }^{9}$ If the projected increase in stereotactic and ultrasound directed biopsies (along with MRI directed biopsies) can be realized, and if we can then combine both Rosen's and Parker's observations, a large number of patients (greater than 33,000) undergoing core directed biopsy of $T_{1 \mathrm{a}}-\mathrm{T}_{1 \mathrm{~b}}$ breast cancer can expect good long-term survivorship. This population would be ideal for interstitial therapy of primary breast cancer.

The microscopic classification of breast cancer is also important. Past series combining both palpable and non-palpable breast cancers report that 80 percent of breast cancers are infiltrating ductal carcinoma. Earlier discovery has generated a shift toward ductal carcinoma in situ. Most stereotactic breast biopsies are performed for a cluster of microcalcifications or small discreet mammographic density. The former is frequently undertaken to rule out ductal carcinoma in situ, while the biopsy of a solid mammographic density, whether by stereotactic or ultrasound guided core, is performed to rule out an infiltrating ductal carcinoma. It is the infiltrating component that presents a risk of local recurrence because of its invasion into the surrounding stroma for a variable distance.

The extent to which $T_{1 \mathrm{a}}-\mathrm{T}_{1 \mathrm{~b}}$ cancers invade tissues, casting cells at some distance from the central nidus, has not been determined, but for large cancers, a correlation exists between the mean diameter of the cancer and the distance that cells are shed from the observable tumor. Holland et al recognized that a 4-centimeter cancer will permeate the stroma farther from its central core than a 2-centimeter breast cancer and that the element of non-visualized ductal carcinoma in situ variably surrounding this core also extends in direct proportion to the tumor size. ${ }^{12}$ This understanding of stromal invasion and extent of ductal carcinoma in situ around the observable cancer is important in determining the extent of interstitial treatment around the observed cancer. Any treatment field should destroy both the observed cancer and its supporting vascular elements (angiogenesis and any vascular invasion), while also treating any cells in the periphery.

Examination of one series of patients undergoing lumpectomy followed by radiation therapy demonstrates that those with cancers of one centimeter or less treated with a 1-centimeter margin followed by a standard course of radiation therapy have a two percent local recurrence rate at six years. ${ }^{13}$ Reflection on both this information and Rosen's observations suggests that early interstitial therapeutic trials must demonstrate rates of cure equal to current therapy. To accomplish this, the treatment field should encompass a biological treatment sphere containing the cancer and a $\mathrm{l}$-centimeter radial margin of normal tissue around it. Thus, the optimal treatment field should be a 3-centimeter diameter in which both the tumor and surrounding stroma are destroyed. In selecting an optimal tumor for treatment, we should exclude cancers will ill-defined domains (e.g., extensive DCIS) and those with a tendency to extend miasmically into the stroma (infiltrating lobular carcinoma). While this may limit the number of patients eligible for trial in the early phases of interstitial therapy, it should insure a low recurrence rate. These demographics and clinical observations are very important in determining the technical requirements of instruments for interstitial therapy of primary breast cancer. 


\section{THERAPEUTIC APPROACH: TREATMENT MODALITY AND IMAGING}

In extending the approach from an imaged core biopsy to the interstitial treatment of a small breast cancer, three elements need to be considered: imaging the biopsied tumor, selection of the treatment approach, and determination of the field of treatment.

Imaging the tumor. Most image directed breast core biopsies are performed using either stereotactic digital mammography or ultrasound. When employing a vacuum-assisted core biopsy, enough tissue may be removed that later recognition of the tumor for treatment is difficult. This problem has been solved in part by placement of a small metallic clip through the 11 gauge biopsy instrument immediately following the procedure. While in theory the presence of metal may pose a problem for the patient undergoing hyperthermic therapy, the clip is small enough that it probably will not present a practical issue. When a 20 millimeter single coring device is employed, most or virtually all of the tumor is removed, again requiring the placement of a marker for subsequent mammographic recognition. Ultrasound directed cores are currently performed with a spring-loaded 14 gauge small core sampling instrument, leaving most of the tumor intact for later rediscovery. ${ }^{14}$ Recently, the vacuumassisted coring device has become commercially available for ultrasonic biopsy; this will no doubt present the same problem in relocation of the tumor as is currently faced mammographically.

Magnetic resonance imagery (MRI) is exquisitely sensitive in defining cancer. Two problems with MRI directed biopsy have limited its use to experimental protocols: 1) the in ability to directly place a biopsy device into an imaged tumor while the patient remains in the magnet, and 2) the ferrous metals in most biopsy instruments cannot be imaged by MRI. Experimental development of a non-metallic vacuum-assisted core biopsy instrument is under investigation.

Selection of the treatment approach. An understanding of the biology of breast cancer is essential in developing a therapeutic strategy. This, in turn, will shape the bioengineering approach to therapy and a determination of the parameters of the treatment field. Three elements are important in this understanding: 1) an interstitial treatment approach wide enough to encompass the tumor and its surrounding stroma; 2) a method of visualizing the tumor so that the treatment elements can be brought to bear against it; and 3) a measurement of the therapeutic field to determine its adequacy.

The current approach is considerably different from the strategy used to develop hyperthermia two decades ago when the goal was to preserve the normal tissue while the cancer cells, considered more heat labile, were to be selectively killed. The present strategy is to destroy the tumor and its surrounding supportive stroma, with the understanding that even a few cancer cells, strayed from the tumor mass, might give rise to locally recurrent disease. Consequently, not only must the treatment field eliminate or destroy the primary observed cancer, but it also must destroy its supporting surrounding stroma in a welldefined, sharply-delineated field at some distance from it. To accomplish this, the sphere of destruction should be non-linear; that is, not a gradual decrease in the treatment area, but one with a sharply-defined field. 
Destruction of the primary breast cancer has been approached through several different means. Coming to basic principles, the tumor must be destroyed either by mechanical removal or by some physical (thermal) approach. Mechanical removal of breast cancer is an extension of the diagnostic image directed biopsy technique to a therapeutic use. The two methods currently employed are removal of a single large volume of tissue en bloc and the milling of the tumor and its surrounding bed by a vacuum-assisted biopsy core sampling device. Both are to some extent industry-driven. The appeal is that the technology is readily at hand and would require little more than the prospective clinical study to determine its efficacy.

The single large coring device (ABBI ${ }^{\circledast}$ produced by U.S. Surgical) deploys a mid-axial single wire under stereotactic guidance to the center of the tumor. Then a 20 millimeter core is taken down the axial guide to encompass the tumor and its surrounding stroma. Of advantage to the pathologist is the ability to examine the cut margin either en face or through multiple slices to measure the distance between the tumor edge and the cut margin. This is very similar to a lumpectomy performed in the operating room. The disadvantages of this approach lie in the initial incision of 20 millimeter size to accommodate the larger coring device. Beyond this, the operator must be certain that the well-defined visualized tumor is centered exactly within the field because only a few millimeters' distance separate the tumor edge and the cut surface. Those cells that have permeated the stroma for varying distances beyond the central tumor nidus may not be captured by such an approach. The development of an even larger device to a 30-millimeter diameter would require an even larger incision, and thus would not cosmetically achieve the goal of an interstitial treatment approach. Because of its availability, current trials are underway at several sites in the United States; there is no currently published data as to their efficacy or the determination of residual disease in the periphery around the treatment site.

Employment of vacuum-assisted core biopsy instruments (Mammotome ${ }^{\circledast}$ by Ethicon Endosurgery and MIBB $^{\circledast}$ [Minimally Invasive Breast Biopsy] by U.S. Surgical) are mammographically dual-chambered core sampling devices that require a single placement of the stainless steel disposable instrument (Figure 1). Suction applied to the lower chamber pulls tissue through a portal into the upper chamber through small holes between the two. The operator then activates the coring device, a hollow cutting tube, that at 1200 RPM is slowly moved along the upper chamber to remove the longitudinal core ( 14 gauge, 11 gauge, and an experimental 3 gauge size), then pulling it back into a chamber outside the breast where the tissue may be extracted and presented for a subsequent radiographic confirmation that the area of concern has been removed. This approach would require a milling program to remove multiple contiguous core samples. The tumor would be excised in pieces. The one advantage of this approach would be the ability to tailor the milling site to an irregularly shaped tumor. At present, the commercially available 11 gauge device has a limited coring field of $1 \frac{1}{2}$ centimeters of aggregate cores. This defines too small a rim around the periphery of a one-centimeter tumor to be assured of total removal of cells beyond the visualized central site.

Both the large and small core instruments have been fitted to stereotactic tables for diagnostic purposes. In issuing approval for these biopsy devices, the Food and Drug Administration has made it quite clear that the instruments are approved for diagnostic use only. A therapeutic extension is currently being evaluated at several sites in the United States for both instruments. In addition, the Mammotome ${ }^{\circledR}$ has been adapted for ultrasonographic 
visualization. A prototype of this instrument is also being constructed of non-ferrous materials that may be visualized through magnetic resonance imagery. A large ( 3 gauge) Mammotome ${ }^{\circledast}$ device is under clinical investigation at several sites in the United States at the present time.

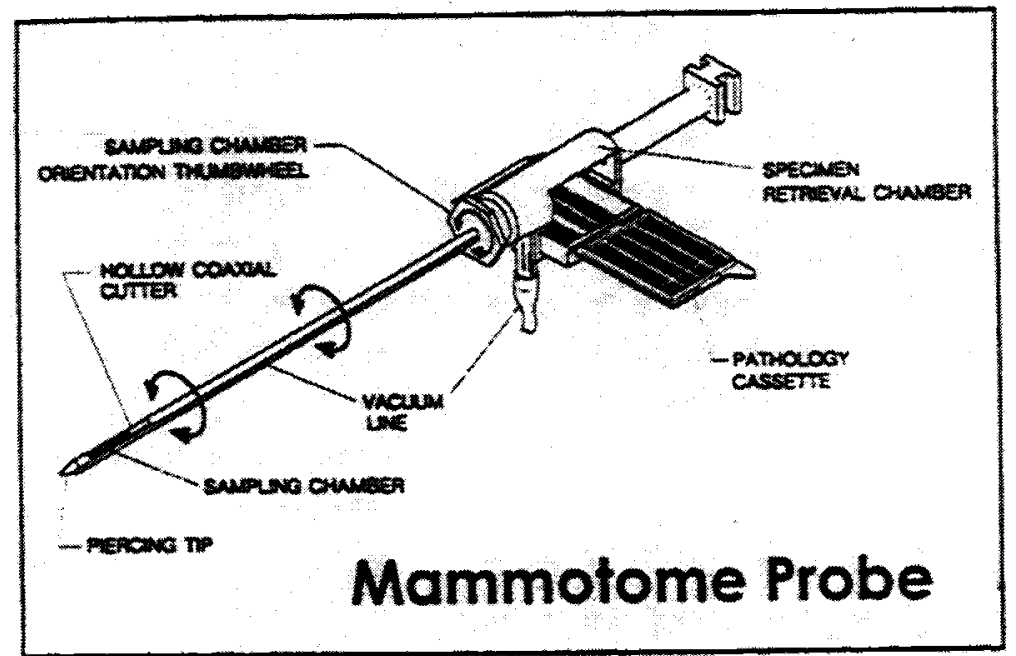

Figure 1: Drawing showing the elements of the Mammotome probe including the piercing tip, the sampling chamber, the hollow coaxial cutter, the vacuum line, the sampling chamber orientation thumbwheel, the specimen retrieval chamber, and the pathology cassette. Different pathology cassettes can be used at various times in the mammotomy procedure to code the location from which specimens were obtained. If a lesion were deposited into one cassette and tissue outside the lesion were deposited into another cassette, it would be possible to determine when the margins of the lesion are free of tumor.

An alternative to removal of the primary breast cancer is treatment in situ. All in situ treatment strategies to date invoke a thermal change out of the physiologic norm to bring about cell death.

Cryotherapy. Cryotherapy, clinically used to treat metastatic disease to the liver, has now been adapted to breast cancer therapy. Rabin et al have reported the development of a cryosurgical technique using a needle through which liquid nitrogen is circulated. ${ }^{15}$ The tissues are cooled from $37^{\circ} \mathrm{C}$ to $-55^{\circ} \mathrm{C}$ within 15 seconds, and with further cooling to a steady state of $-140^{\circ} \mathrm{C}$ at over two minutes. The average diameter of the ice ball grows to be 22.3 millimeters within five minutes in in vivo studies of sheep breast tissue. The ice ball is imaged through ultrasound analysis; the mechanism of injury is thought to be membrane disruption during the freeze/thaw cycle. The only human trial to treat primary breast cancer was reported in 1997 by Staren et al. ${ }^{16}$ The study demonstrates feasibility in a small number of patients with short-term follow-up. 
Of practical concern is that the dimensions of the ice ball are sometimes difficult to. determine on the ultrasound image. This tends to underestimate the size of the treatment field. A theoretical concern is that not all of the tumor cells may be killed by this process. In dealing with metastatic disease to the liver, failure seems far less crucial when there is an expectation that the disease will progress to new sites yet unseen. Treatment failure of a single site demonstrated by regrowth of cancer within the treatment field is far less critical than regrowth within the treatment field of a small human primary breast cancer that could otherwise be completely cured through excision followed by adjuvant therapy. Further evidence of efficacy is needed before a long-term clinical trial should be entertained.

Hyperthermia offers the more common approach to interstitial therapy. The addition of energy through one of several modes will be translated to heat that in turn will cause denaturation of proteins, with eventual cell death. Several technologies are now under investigation that will provide heat. They include microwave, ultrasound, radiofrequency, and laser.

Microwave. Fenn and his colleagues at MIT have described an adaptive microwave phased array that will target tumors deep within the intact breast. Animal studies were reported in 1999. ${ }^{17}$ A company, the Celison Corporation, has been formed to produce this technology in the treatment of breast and prostate cancer. A current prototype is under evaluation at two sites in the United States. Temperature fields of three to five centimeters heated up to $46^{\circ}$ $\mathrm{C}$ for ten minutes define the treatment pattern. The efficacy and clinical importance of this remain to be demonstrated.

Radiofrequency. Hyperthermic ablation by radiofrequency occurs when a high-frequency alternating current is administered to tissue, causing motion of intracellular ions that results in intracellular thermal heating by friction. This is similar to the electrocautery used in the operating room, which operates on a similar principle to bring about dessication (Figure 2).

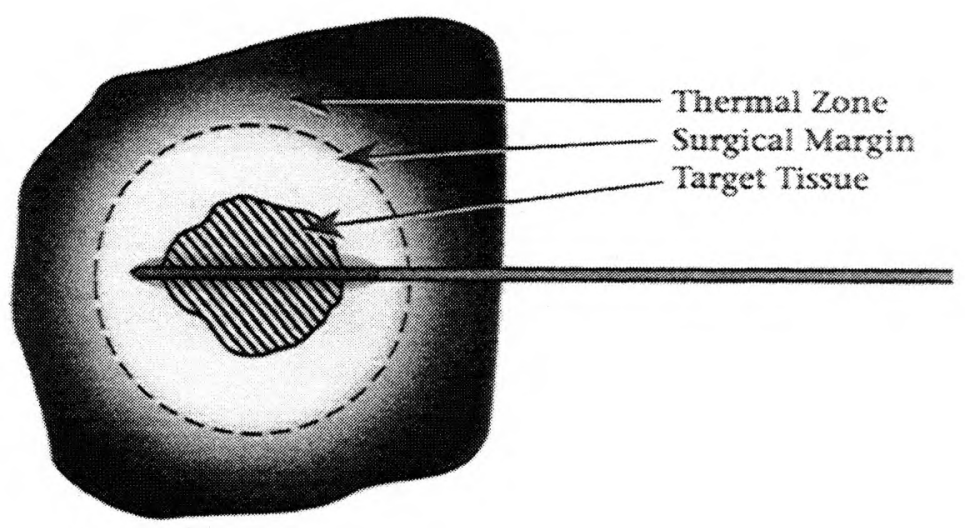

Figure 2: Cool-tip RF

McGhan et al of the University of California at Davis recently reported an animal model of radiofrequency ablation of breast tissue. ${ }^{18}$ Jeffrey and her colleagues at Stanford and U.C. 
Davis reported the first radiofrequency ablation of human breast cancer in October of $1999 . .^{19}$ In this study, five women with locally advanced invasive cancer underwent radiofrequency treatment prior to surgical resection of the tumor itself. This was accomplished under general anesthesia with a 15 gauge insulated multiple needle electrode placed under guidance. Low power radiofrequency was administered over a 3-minute period. Three of the five patients demonstrated heterogeneity in the pattern of necrosis, while two demonstrated complete cell death of the tumor. The authors reported that a zone of ablation extended for a diameter of 0.8 to 1.8 centimeters around the tumor. This feasibility study incorporating ultrasound imagery will require additional documentation to demonstrate a sustained high level of efficacy. Another early prospective analysis has been initiated at the M.D. Anderson Hospital in Houston, Texas, with results far too early to comment upon. ${ }^{210}$

Use of radiofrequency is attractive in that it is familiar to most surgeons. The sphere of tissue injury and cell kill is currently defined by ultrasound. Because of the nature of the energy source, and because most of the probes are of a ferrous metallic material, use of magnetic resonance imagery is not currently possible to define the treatment field. No doubt a great deal more will be forthcoming in a short time in this area of interstitial hyperthermic therapy.

Ultrasound. Ultrasound may be employed as a hyperthermic therapeutic modality in the treatment of breast cancer. Harari et al in 1991 described the development of a scanned focus approach to ultrasound hyperthermia, ${ }^{21}$ and Hill in 1995 suggested that high-intensity focused ultrasound might be used in the treatment of cancer. ${ }^{22}$ In a recent presentation, Gianfelice et al described magnetic resonance focused ultrasound ablation of primary breast cancer in patients judged to be poor operative candidates. ${ }^{23}$ In evaluating 15 patients, the authors report that everyone underwent post-treatment MR imaging revealing a hypointense rim that partially surrounded the breast treatment field. This was presumed to represent tissue destruction.

The most eloquent of ultrasound treatment proposals has been reported by Lu $\mathrm{et} \mathrm{al} \mathrm{from} \mathrm{the}$ Joint Center for Radiation Therapy at Harvard Medical School. ${ }^{24}$ The authors note that ultrasound is capable of penetrating deep into tissues. The device is a cylinder comprised of a stack of rings, each ring containing 48 transducers mounted on the inside of the ring and directed toward the center. The transducers operate in one of two frequency bands (1.8-2.8 $\mathrm{MHz}$ and 4.3-4.8 MHz) arranged alternately in each ring. In the course of treatment, patients were placed prone with the breast passed through the table and immersed in water surrounded by the cylinder. Using a simulator, the authors demonstrated a system capable of achieving and maintaining a temperature distribution of $41.5^{\circ}-44^{\circ} \mathrm{C}$ in a quadrant of the breast. This approach, initially designed for the treatment of intraductal carcinoma, is in early clinical trial awaiting confirmation.

Laser. Laser therapy in the interstitial treatment of breast cancer has caught the imagination of several investigators. Dowlatshahi was the first to report necrosis of a mammary tumor by interstitial Nd:YAG laser hyperthermia in a rat model in $1992,{ }^{25}$ and he was the first to report a feasibility study using stereotactically guided interstitial laser therapy at the SPIE meeting in $1997 .{ }^{26}$ Our laboratory has generated significant interest in the development of this biotechnology and light dispersion through several publications..$^{27,28,29,30,31}$

Temperature increases from laser interstitial therapy (LTTT) bring about cell necrosis through the heating of the target tumor by means of a laser source (Figure 3). Lasers 
typically used for this process are continuous wave near-infrared lasers such as the Nd:YAG laser $(1064 \mathrm{~nm})$ or an array of diode lasers $(808-980 \mathrm{~nm})$. Heat is generated from light scatter by means of laser energy delivered through the fiberoptic probe that is inserted into the tissue under x-ray, MRI, or ultrasonic guidance. The treatment parameters (power, duration, and wave length) suitable for any LITT procedure are often developed through thermo-optical models that will predict a laser-induced temperature elevation within the specific organ or tissue to be treated. Much of the work in our laboratory has been accomplished through use of Nd:YAG lasers. In developing a model of thermal response, we placed an array of copper-constan thermocouples at fixed intervals through a plexiglass grid with holes placed in a square pattern at 5-millimeter intervals (Figure 4). These were arrayed so that the depth of the needle came to the plane of the probe. In time, the study of tissue samples of human and porcine tissue ex vivo and in vivo have permitted the development of thermal models.

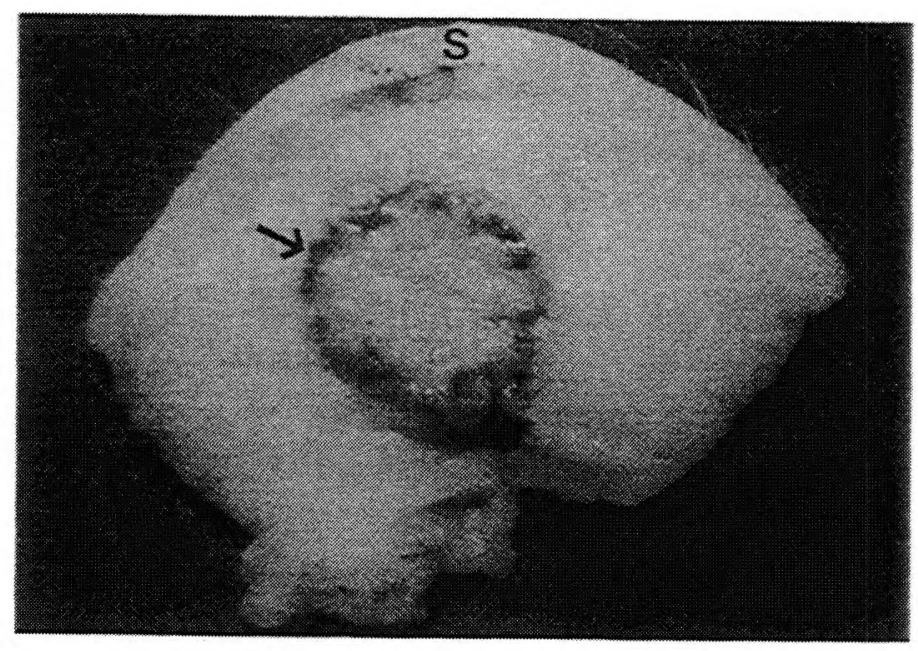

Figure 3: Macroscopic tissue specimen of in vivo porcine subcutaneous fat and overlying skin taken 27 days after laser treatment. Arrows mark the rim of transition. S, skin.

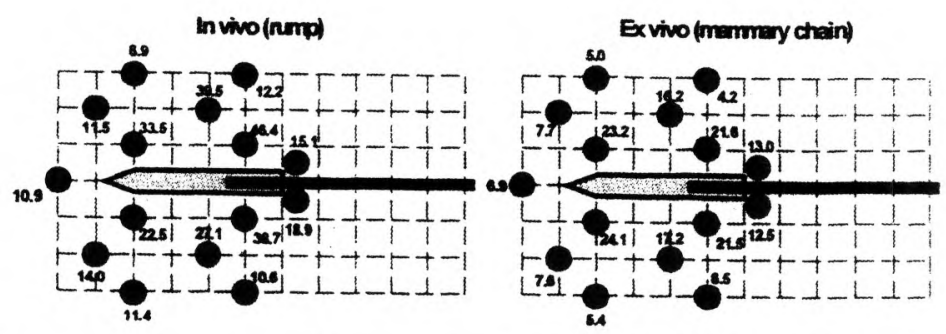

Figure 4: Temperature increase produced in porcine fatty tissue in vivo (left) and ex vivo (right) after one LPS treatment cycle. 
There is no constitutive animal model for the human breast, but it now recognized that involution with menopause in patients not receiving supplemental exogenous estrogen will permit the breast tissue to become approximately $85 \%$ fat. Consequently, an alysis of fat in terms of light scatter and heat generation seems like a good place to begin. Ideally, in this form of hyperthermia and in any other in which an interstitial probe is placed, there should be a good even distribution of the energy field that will translate to a uniform distribution of hyperthermia and consequent tumor necrosis. At the same time, one should avoid charring of the fiber that will alter the uniform output of light energy and of overheating leading to bubble formation, again producing irregularities in the thermal distribution.

It is our hypothesis that all of the cells within the treatment field should be killed, including the surrounding stroma, to prevent nurturing even one or two breast cancer cells beyond the central nidus of the tumor itself. To achieve that end, tissues near the probe were heated to over $50^{\circ} \mathrm{C}$, while tissues at a distance of 1.5 centimeters may have only had an elevation of $5^{\circ}$ to $6^{\circ} \mathrm{C}$ (Figure 4). If the response of breast cancer is as we understand it, then the center of the probe should be placed within a one-centimeter tumor, with a strategy to deliver energy to create a 1.5-centimeter radial zone of sharply-defined tissue denaturation and cell death. Using the Nd:YAG laser, it was our good fortune to produce oval areas of focal kill within subcutaneous in vivo porcine fat that had a diameter of between 2.8-3.1 centimeters (Figure 3). This was rimmed by a 1-2 millimeter ring of color change that early on represented thrombosis, and later a focus of granulation tissue and repair. Beyond that, the tissues appeared to be unchanged by the hyperthermic event.

It is important that such a treatment program, regardless of whether it be induced by laser, radiofrequency, or any of the other approaches to interstitial therapy, produce a uniform field of treatment that is sharply defined and broad enough to encompass not only the cancer, but its surrounding peripheral supportive stroma. Moreover, such a treatment plan should be highly reproducible in both phantom studies, ex vivo tissue, and in an in vivo setting. After all, the margin permitted for error is virtually zero when it comes to a long-term clinical trial for the breast cancer patient. Furthermore, to be truly successful, such a program should have minimal or no surface change on the breast skin and subcutaneous tissue. This again will require a patient selection to be certain that tumors are deep enough that the thermal field will not denature those tissues close to the skin.

Measurement of the therapeutic field. It is important to determine the therapeutic field both in its change during the course of therapy and in its volume. Changes are often measured by temperature monitored by thermocouples (Figure 4 ) or by fiberoptic thermal sensors. The shape and dimension of the thermal field can best be determined through imaging. Images are generated through ultrasound, mammography or magnetic resonance imaging. Ultrasound can be effective in measuring the ice ball in cryotherapy, and it may well demonstrate the dimensions of tissue denaturation in hyperthermia. It is a mainstay of radiofrequency probe placement and tumor evaluation. With technological advances, the ability of ultrasound to resolve subtle differences may be enhanced. Clearly, it will remain central in the armamentarium of the interstitial therapist in breast disease.

One of the most exquisite and sensitive approaches to imaging the breast tumor and its response to hyperthermia is magnetic resonance imagery. By design, it can produce threedimensional images and can be used in almost real time. As observed earlier in this review, biopsy needle and therapeutic probe placement are not trivial tasks in that the patient must 
be marked, brought from the magnet without moving, have the treatment probe placed, and then replaced once again in the magnet. Moreover, anything that has a ferrous metallic component to it cannot be used with MRI; therefore, radiofrequency, partly by the nature of its energy source and partly because of its physical make-up, is inappropriate for use with MRI. This would also apply to cryotherapy. The laser interstitial probe, on the other hand, is entirely non-metallic and should be easily visualized by magnetic resonance imagery. Finally, beyond the current technical difficulties imposed by MRI, cost and FDA approval for breast imaging remain issues to be reckoned with. Still, this modality may be the most exquisite approach to imaging of hyperthermic change of all the available possibilities.

With the transition from analog $\mathrm{x}$-ray to charge couple device screen, digital mammography became an integral part of the stereotactic breast biopsy instrument. Our laboratory is currently evaluating quantitative changes in grayscale difference with thermal alteration. Two issues face such an investigation: the decrease in density with the warming of tissues that will allow $x$-rays to better penetrate the plate, thus making the image darker, and at the same time, gradual denaturation of tissues that will become more dense, thus impeding $x$ rays' arrival at the plate, making the image lighter. Analyses of both of these through study on tissue phantoms should eventually lead to a non-invasive quantitative determination of both temperature and denaturation that will demonstrate when the tissue field in question has been adequately treated. Much work remains to be done in this domain.

\section{PROBLEMS TO BE SOLVED}

Regardless of the approach taken in the interstitial therapy of breast cancer, clinical trials must be developed to examine the efficacy of that program in the treatment of human disease. Initially, such trials should be highly selective, based on tumor criteria and the expected compliance of the patient to remain on study. Early studies no doubt will involve treatment programs prior to lumpectomy or mastectomy so that the tumor tissue and its surrounding stroma may be examined carefully to determine the efficacy of treatment. Ultimately, patients will not undergo surgical removal of the tumor and will be followed probably with magnetic resonance imagery, mammography (because of its lower cost), and intermittent interventional core biopsy. Long-term studies will be needed at both alpha and beta sites to justify this paradigm shift from excision to interstitial therapy.

In evaluating the tissues treated, a better histologic marker than Haematoxylin and Eosin needs to be employed. Hyperthermic cell injury causes nucleolysis that can only be seen, at the earliest, 72 hours after injury by $\mathrm{H} \& \mathrm{E}$. If early trials are to succeed, then patients should be treated with some immediacy. A PCNA marker has been used by Mumtaz and Harms, while Jeffrey and her co-authors have suggested a stain for NADH-diaphorase. The most appropriate marker remains to be determined.

\section{SUMMARY}

This review has evaluated both the demographic and biological characteristics of breast cancer patients and their diseases. We have explored several approaches to interstitial therapy and have presented some of the problems yet to be resolved before interstitial therapy of breast cancer can be routinely employed. 


\section{REFERENCES}

1. Halsted WS. The results of operations for the cure of cancer of the breast performed at the Johns Hopkins Hospital from June, 1889 to January 1894. Johns Hopkins Hospital Bulletin 1894-95; 4:297-321.

2. Shapiro $S$, Strax $P$, Venet $L$. Periodic breast cancer screening in reducing mortality from breast cancer. JAMA 1971;215:1777-85.

3. Fisher B, Bauer M, Margolese R, et al. Five-year results of a randomized clinical trial comparing total mastectomy and segmental mastectomy with or without radiation in the treatment of breast cancer. N Eng J Med 1985; 312:666-73.

4. Moffat FL. Locoregional treatment and considerations in early breast cancer. R.G. Landes Company, Austin, Texas, 1994.

5. Blomgren J, Jacobson B, Nordenstrom B. Stereotactic instrument for needle biopsy of the mamma. Am J Radiol 1977; 129:121-25.

6. Dowlatshahi K, Yaremko ML, Kluskens LF, et al. Nonpalpable breast lesions: Findings of stereotaxic needle-core biopsy and fine-needle aspiration cytology. Radiology 1991; 181:745-50.

7. Jackson VP, Reynolds HE. Stereotaxic needle-core biopsy and fine-needle aspiration cytologic evaluation of nonpalpable breast lesions. Radiology 1991; 181:633-34.

8. Sterrett G, Oliver D, Frayne J, et al. Stereotactic fine needle aspiration biopsy (SFNB) of breast: Preliminary results in Perth with the TRC Mammotest machine. Cytological aspects. Pathology 1991; 23:302-10.

9. Parker SH, Burbank F, Jackman R, et al. Percutaneous large core breast biopsy: A multiinstitutional study. Radiology 1994; 193:359-64.

10. Cady B, Stone MD, Schuler JG, et al. The new era in breast cancer. Arch Surg 1996; 131:301-08.

11. Rosen PP, Groshen S, Kinne DW, et al. Factors influencing prognosis in node negative breast carcinoma: Analysis of $676 \mathrm{~T}_{1} \mathrm{~N}_{0} \mathrm{M}_{0} / \mathrm{T}_{2} \mathrm{~N}_{0} \mathrm{M}_{0}$ patients with long term follow-up. J Clin Oncol 1993; 11:2090-2100.

12. Holland $R$, Veling $S H$, Mruvunac $M$, et al. Histologic multifocality of $T_{\text {is }}$ and $T_{1-2}$ breast carcinomas. Implications for clinical trials of breast-conserving surgery. Cancer $1985 ; 56: 979$ 990.

13. Barterlink H, Borger JH, van Dongen JA, et al. The impact of tumor size and histology on local control after breast-conserving therapy. Radiotherapy and Oncology 1988; 11:297-303.

14. Staren ED, Fine R. Breast ultrasound for surgeons. Am Surgeon 1996; 62:108-12.

15. Rabin $\mathrm{Y}$, Julian TB, Olson $\mathrm{P}$, et al. Development of a cryosurgical technique for breast malignancies. Proceedings SPIE 1999; 3590:465-71.

16. Staren ED, Sabel MS, Gianakakis LM, et al. Cryosurgry of breast cancer. Arch Surg 1997; 132:28-33.

17. Fenn AJ, Wolf GL, Fogle RM. An adaptive microwave phased array for targeted heating of deep tumors in intact breast: Animal study results. Int J Hyperthermia 1999; 15:45-61.

18. McGahan JP, Griffey SM, Schneider PD, et al. Radiofrequency ablation of breast tissue in the animal model. RSNA Scientific Program, Abstract 27. Breast Intervention, Chicago, IL, November 28, 1999.

19. Jeffrey SS, Birdwell RL, Ikeda DM, et al. Radiofrequency ablation of breast cancer. Arch Surg $1999 ; 134: 1064-68$.

20. Singletary E. Personal communication.

21. Harari PM, Hynynen KH, Roemer RB, et al. Development of scanned focused ultrasound hyperthermia: Clinical response evaluation. Int J Radiat Oncol Biol Phys 1991; 21:831-40.

22. Hill CR, ter Haar GR. High intensity focused ultrasound-potential for cancer treatment. Br J Radiol 1995; 68:1296-1303.

23. Gianfelice DC, Mallovche H, Lepanto L, et al. MR-guided focused ultrasound ablation of primary breast neoplasms: Works in progress. RSNA Scientific Program, Abstract 25. Breast Intervention, Chicago, IL, November 28, 1999.

24. Lu X-Q, Burdette EC, Bornstein BA, Hansen JL, et al. Design of an ultrasonic therapy for breast treatment. Int J Hyperthermia 1996; 12:375-99. 
25. Dowlatshahi K. Babich D, Bangert J, Kluiber R. Histologic evaluation of rat mammary tumor necrosis by interstitial Nd:YAG laser hyperthermia. Lasers Surg Med 1992; 12:159-64.

26. Dowlatshahi K, Fon M, Shekarklau BA, et al. Stereotactic interstitial laser therapy for early-stage breast cancer. Breast Jol 1996; 2:304-11.

27. Robinson DS, Parel JM, Denham DB, Manns F, Gonzalez X, Schachner R, Herron A, Burdette EC. Stereotactic uses beyond core biopsy: Model development for minimally invasive treatment of breast cancer through interstitial laser hyperthermia. American Surgeon 1996; 62:117-18.

28. Robinson DS, Parel JM, Denham DB, Gonzalez-Cirre X, Manns F, Milne PJ, Schachner RD, Herron AJ, Commander J, Hauptmann G. Interstitial laser hyperthermia model development for minimally invasive therapy of breast carcinoma. Journal of the American College of Surgeons 1998; 186:284-92.

29. Robinson DS, Parel JM, Denham DB, Manns F, Gonzalez X, Schachner R, Herron A, Burdette EC. Model development of laser fiberoptic endoablative treatment for primary breast cancer. Proceedings SPIE 1996; 2671:142-45.

30. Manns F. Milne PJ, Gonzalez-Cirre X, Denham DB, Parel JM, Robinson DS. In-situ temperature measurements with thermocouple probes during laser interstitial thermotherapy (LITT): Quantification of a measurement artifact. Lasers in Surgery and Medicine 1998; 23:94-103.

31. Milne PJ, Manns F, Denham DB, et al. In-situ measurement and analysis of the temperature fields in laser interstitial thermotherapy of breast cancer. Proceedings SPIE 1999; 3590:455 64. 